\title{
Introducing Androgens: Clinical Research and Therapeutics
}

Abdulmaged Traish and Abraham Morgentaler

Welcome to Androgens: Clinical Research and Therapeutics, the official journal of the Androgen Society. Although the effects of testosterone deficiency have been recognized for millennia and testosterone therapy has been available for over 80 years, Androgens is the first journal to bring together all aspects of research and clinical experiences from every corner of the medical world, geographically and biologically.

The last three decades have borne witness to considerable advances in the clinical realm of testosterone deficiency and its treatment and provided a new foundation to build upon clinical advances. This is an exciting time to be fully engaged in the evolving scientific and clinical advances in this health-related medical field. I am very excited to undertake the role of Editor-in-Chief of this new journal alongside my colleague Dr. Morgentaler as the Senior Editor. Our vision is to make Androgens: Clinical Research and Therapeutics an indispensable, inspiring, and trusted source of high-quality scientific knowledge for all disciplines involving androgen deficiency and its therapeutic modalities.

To achieve this goal, we will publish original basic and clinical research, reviews, opinion pieces, roundtable discussions, debates, and other scientific and educational materials that support this evolving field of medicine. Whereas previously one could find testosterone-related articles scattered throughout an enormous variety of journals, usually based on the medical specialty of the authors, we intend for Androgens to become the most highly valued "publication destination" for researchers in the field, regardless of medical discipline, and a reliable platform for interested readers to find a wide range of articles addressing various aspects of testosterone deficiency and its treatment.
We welcome articles on the effects of testosterone deficiency and testosterone therapy on topics encompassing, but not limited to:

- Sexual function and dysfunction

- Prostate diseases

- Muscle growth and function

- Bone health

- Lipid metabolism

- Vascular health

- Anemia

- Sarcopenia

- Diabetes

- Obesity

- Ocular function

- Cognitive function

- Motivation and behavior

- Mood

- Public health and public policy

We also welcome manuscripts that address clinical effects of related hormones and metabolites, such as estradiol and $5 \alpha$-dihydrotestosterone and dehydroepiandrosterone, and we will consider preclinical in vitro and animal studies if they appear to have clear implications for human biology. Androgens aims to publish the highest-quality articles from all disciplines. The broad scope of the journal along with its Open Access format, with Mary Ann Liebert, Inc.'s commitment to promoting research, will ensure the highest potential exposure of the published material. No subscription is required to access the content of this journal, and authors will retain copyrights to their own work. We hope that readers will find this new journal of great value as we work together to usher in a new era of scholarship and collaboration.

(c) Abdulmaged Traish and Abraham Morgentaler 2020; Published by Mary Ann Liebert, Inc. This Open Access article is distributed under the terms of the Creative Commons License (http://creativecommons.org/licenses/by/4.0), which permits unrestricted use, distribution, and reproduction in any medium, provided the original work is properly cited. 
We call on researchers and clinicians with interest in this rapidly advancing field of testosterone therapy to help support this new fledgling journal and to help us realize the overarching goal of bringing clinical research on testosterone deficiency and testosterone therapy into one highly valued resource, that is "Androgens." The editorial team will work tirelessly and diligently with all authors to ensure successful submissions via a fair and amicable review process, with the ultimate goal of expediting publications that advance science in the field.

\section{Abdulmaged Traish, Editor-in-Chief} Abraham Morgentaler, Senior Editor

Cite this article as: Traish A, Morgentaler A (2020) Introducing Androgens: Clinical Research and Therapeutics, Androgens: Clinical Research and Therapeutics 1.1, 1-2, DOI: 10.1089/

andro.2020.28999.atr
Androgens

Clinical Research and Therapeutics 